\title{
Psychothérapies en psycho-oncologie : éthique, technique, évaluation et remboursement des psychothérapies
}

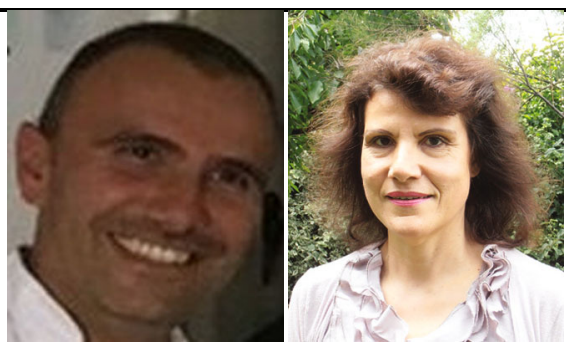

\section{Psychotherapy within Psycho-Oncology Services: Ethics, Techniques, Evaluation and the Reimbursement of Psychotherapy}

\author{
P. Cannone - M.-F. Bacqué \\ (C) Lavoisier SAS 2019
}

Notre idée était de réaliser un état des lieux des interventions psychothérapeutiques en oncologie en France. Quel que soit le modèle utilisé, nous souhaitons mieux appréhender les indications, les problématiques et l'évolution des théories comme des techniques. Nous avons privilégié les principaux paradigmes utilisés en France autour de l'enfant, de l'adolescent et de l'adulte.

Profitons de cet éditorial pour lancer un appel à partager nos pratiques et nos connaissances, dans une visée thérapeutique et pédagogique. Un prochain congrès de la SFPO sur l'état des lieux des psychothérapies en oncologie pourrait être l'occasion d'échanger plus directement entre les courants. Ainsi, les thérapies à médiation ouvrent la clinique en montrant un changement de posture et une augmentation des configurations thérapeutiques. Des psychologues utilisent le dessin avec les adultes, d'autres des haïkus ou de la poésie, certains proposent de visionner un court métrage sur les expériences de mort imminente retravaillé en entretien individuel ou groupal. Ces initiatives montrent une véritable évolution ; à critiquer puis à diffuser...

\section{Respecter les patients en besoin de psychothérapie et les psychothérapeutes}

Respect, c'est le mot qui vient spontanément, parce que trop souvent, nous avons été confrontés aux guerres idéologiques

P. Cannone $(\bowtie)$

Service d'oncologie multidisciplinaire

et innovations thérapeutiques,

hôpital Nord, AP-HM, chemin des Bourrely,

F-13915 Marseille cedex 20, France

e-mail : patrice.cannone@ap-hm.fr

M.-F. Bacqué ( $\square)$

SuLiSoM, université de Strasbourg,

12, rue Goethe, F-67000 Strasbourg, France

e-mail : mfbacque@club-internet.fr qui desservent encore le débat entre psychistes. Ici l'ensemble des articles questionne, critique et propose des échanges qui prennent en considération les cadres des autres praticiens ou paradigmes. La psycho-oncologie pourrait être cette voix qui rassemble la diversité thérapeutique pour accompagner l'évolution de la société.

Respect, c'est aussi et surtout respecter la clinique des praticiens, dans ce lieu (l'hôpital) et ce temps (la maladie) de la démesure, aux limites du corps, aux limites de la douleur, aux limites du supportable, telle une forme d'excès des affects et de violence des mots et des gestes. Cette clinique, aussi riche que dévorante, intime de la borner, d'y joindre en permanence notre déontologie, l'étayage théorique et le travail d'analyse personnelle de tout thérapeute.

Respecter la clinique des praticiens, c'est respecter le temps psychique pour l'autre comme pour soi, le temps de la lecture, de l'écriture et de la parole. Respecter c'est aussi et surtout résister aux assauts de l'accélération d'un temps productif et efficace, résister à l'uniformisation des pratiques sans nuances ni reliefs prises dans les filets des comptabilités glacées des institutions.

\section{Place des psychothérapies dans le paysage médical français}

Les psychothérapies sont plus que jamais d'actualité en France. Ainsi, le VIII ${ }^{\mathrm{e}}$ Congrès mondial des psychothérapies s'est déroulé à Paris en 2017 avec pour thème « Paix, Empathie et Psychothérapie » pour délivrer un message d'humilité et de respect de nos pratiques, confrontées à l'étendue des souffrances psychiques. Au-delà des divergences théoriques des modèles psychothérapiques ou des comparaisons des apports et limites de tel ou tel paradigme, nous pouvons au moins définir une éthique commune, questionner la place d'un symptôme psychique secondaire ou préexistant à une 
pathologie somatique et rester ouverts aux sources historiques de l'expérience psychothérapeutique.

Par exemple, sommes-nous prêts à réfléchir aux grandes questions comme celle du remboursement des psychothérapies ? Il semble juste que les psychothérapies soient remboursées parce que leurs effets thérapeutiques sont prouvés lorsqu'on les confronte à une liste d'attente ou à l'absence de traitement et, qui plus est, parce qu'elles ont de meilleurs résultats à long terme que les traitements médicaux les plus efficaces [1]. L'épidémiologie des troubles mentaux montre que la maladie psychiatrique aiguë ou chronique touche les catégories socio-économiques les plus faibles. L'idée est donc de favoriser l'accès de tous les assurés à la psychothérapie auprès de psychologues cliniciens ou psychothérapeutes agréés par l'ARS (Agence régionale de santé) grâce à un remboursement par la sécurité sociale ${ }^{1}$. Même si la démarche paraît séduisante, le dispositif expérimental comporte, à notre sens, de nombreux points d'achoppements. Tout d'abord, le groupe à l'origine du dispositif a invité un représentant du SNP (Syndicat National des Psychologues) sans tenir compte des revendications explicites issues de nombreuses pétitions des professionnels. Le SNP a donc été inclus, mais sans être entendu, ce qui a donné l'impression qu'il validait le projet alors qu'il tentait de trouver d'autres issues. L'AFTCC (Association Française de Thérapie Comportementale et Cognitive) a également participé, mais nous verrons plus loin qu'elle s'exprime aujourd'hui largement contre la proposition finale. Le Collège de la médecine générale, le Conseil national de la psychiatrie et le Collège national pour la qualité des soins en psychiatrie ont signé ce guide pratique à destination des psychologues cliniciens et des psychothérapeutes.

Trois départements (Bouches-du-Rhône, Haute-Garonne et Morbihan) s'inscrivent dans l'expérimentation de la Sécurité sociale prévue de 2017 à 2020 auprès des 18-60 ans. Le critère d'inclusion est « un syndrome dépressif ou anxieux, des troubles de l'adaptation ou des troubles médicaux inexpliqués » en reprenant la classification de la CIM-10. L'idée du remboursement trouve son origine dans le record battu par la France en termes de consommation de psychotropes. La HAS (Haute Autorité de santé) a préconisé depuis 2017 la psychothérapie en première intention dans la prise en soin de l'épisode dépressif caractérisé, d'intensité légère ou modérée [2]. Bien sûr, le risque suicidaire et les symptômes somatiques sont évalués par le médecin. Mais ici, la psychothérapie peut être réalisée indifféremment par le médecin généraliste, le psychologue ou le psychiatre. Si le dispositif expérimental propose le remboursement des psychothérapies à des non-

\footnotetext{
${ }^{1}$ Guide pratique à destination des psychologues cliniciens et des psychothérapeutes. Prise en charge par l'assurance maladie des thérapies non médicamenteuses. Dispositif expérimenté dans 3 départements.
}

médecins (les psychologues), il le propose aussi à d'autres praticiens que les psychologues ; les médecins, avec toutefois une grande différence, celle de la qualification. Côté médecine, les psychiatres et médecins généralistes ont leur propre cotation de leurs actes, non spécifiée « psychothérapie ». Les psychologues auraient, pour la première fois, leur acte psychothérapique coté (les bilans psychologiques le sont déjà). En revanche, la psychothérapie pratiquée par le médecin généraliste pose la question de sa formation et du cadre de son exercice (peut-on dans le même cadre de travail exercer la médecine et la psychothérapie ?), par ailleurs, le temps de consultation peut-il être respecté par le médecin (at-il le temps pour une séance d'a minima trente minutes alors que sa salle d'attente est pleine ?). Enfin si la formation à la psychothérapie est clairement l'objet des masters de psychologie clinique, y a-t-il une formation à la psychothérapie dans les études médicales? Et dans les DES de psychiatrie ${ }^{2}$, la formation de psychothérapeute est-elle explicitement nommée et réalisée?

\section{Comment fonctionne le remboursement expérimental des psychothérapies depuis juillet 2017 dans trois départements français ?}

Tout repose sur le médecin généraliste qui rencontre un patient anxiodépressif et prescrit un entretien d'évaluation tarifé $32 €$ pour 45 minutes auprès d'un psychologue clinicien (ou d'un psychothérapeute ou du médecin généraliste). Quelle est alors la formation des médecins généralistes sur les psychothérapies disponibles et leur proposition selon le type de pathologies rencontrées ? Les indications diagnostiques sont fondées uniquement sur deux échelles, le PHQ- $9^{3}$ et la GAD- $7^{4}$. Le psychologue intervient dans les dépressions légères à modérément sévères et dans le cas d'anxiété légère à modérée. La valorisation financière des compétences du psychologue-psychothérapeute est fixée au tarif de $32 €$ l'entretien

\footnotetext{
${ }^{2}$ L'arrêté du 21 avril 2017 relatif aux connaissances, aux compétences et aux maquettes de formation des diplômes d'études spécialisées, fixe la liste de ces diplômes, options et formations spécialisées transversales du troisième cycle des études de médecine. Le Journal officiel du 28 avril 2017 demande aux futurs psychiatres de "proposer le suivi psychothérapeutique le plus approprié ». Il s'agit d'une compétence visée au cours du DES de psychiatrie. Mais que la réalisation de la psychothérapie soit l'œuvre du psychiatre en formation initiale n'est absolument pas explicite (que ce soit en phase socle, d'approfondissement ou en phase de consolidation).

${ }^{3}$ Le Patient Health Questionnaire en neuf items.

${ }^{4}$ L'échelle Generalized Anxiety Disorder-7 a une version française validée comme pertinente pour le dépistage du trouble anxieux généralisé chez les patients avec une épilepsie. Spitzer et al., Arch Intern Med, 2006 et Micoulaud-Franchi et al., Epilepsy and Behavior, 2016.
} 
d'évaluation puis $22 €$ les séances de psychothérapie de soutien de 30 minutes, au nombre de dix maximum renouvelables. Une fois les dix séances réalisées, le médecin généraliste ou le psychiatre ont la possibilité en cas d'absence d'amélioration de prescrire dix séances supplémentaires de psychothérapie structurée à $32 €$ la séance. L'évaluation de ce dispositif s'effectue encore par la cotation des deux échelles PHQ-9 et GAD-7, dont les scores orientent le rythme des séances et sont censés « aider » le psychologue.

Le tarif de la consultation psychologique est évidemment choquant de plusieurs points de vue :

- économiquement, une fois les charges sociales payées, il ne reste plus au psychologue qu'environ un peu plus d'une dizaine d'euros pour une séance de psychothérapie et une quinzaine pour l'entretien d'évaluation ;

- des psychothérapies à deux vitesses vont voir le jour. Pourquoi, en effet, les psychiatres psychothérapeutes (en secteur 1) pratiqueraient-ils la psychothérapie à un tarif de $46,70 €$ (leur tarif de consultation) ? Et pourquoi les psychiatres en secteur 2 (honoraires libres) pourraient-ils dépasser les tarifs de la Sécurité sociale alors que les psychologues ne pourraient en aucun cas le faire ?

- ce remboursement très faible des psychothérapies des psychologues et ces interventions nombreuses des médecins (généraliste et psychiatre) dans la prescription et le contrôle des psychothérapies traduisent à nouveau le pouvoir médical sur les plans historique et politique. Ils conservent ici leur monopole des soins, leur autonomie dans leur pratique et une position hiérarchique dominante sur les psychologues et les psychothérapies ;

- enfin, ce tarif dérisoire du paiement des psychothérapies aux psychologues s'explique par un calcul qui permettrait aux hôpitaux de coter enfin les actes psychologiques et de faire « rapporter » le travail des psychologues hospitaliers. Or, ce faible salaire est aussi représentatif de la précarisation massive des psychologues de la Fonction Publique hospitalière (aucun nouveau contrat à durée indéterminée, des temps partiels uniquement, des salaires non revalorisés depuis 25 ans). Cela pousse les patients à prendre l'initiative de rencontrer des psychologues libéraux et donc à externaliser leur demande. Or, le manque de psychologues est cruel dans toutes les structures du secteur psychiatrique? particulièrement dans les Centres Médicaux Psychologiques (CMP).

Dernier point, bien avant que la Sécurité sociale ne se préoccupe du remboursement, les psychologues ont toujours reçu des patients. Or à chaque fois, ils élaborent un diagnostic de fonctionnement psychique, prennent en compte les médications, le risque suicidaire, mettent en place des bilans psychologiques, analysent l'évolution de leur traitement (entretiens, psychothérapie de soutien ou structurée et souvent passage de l'une à l'autre), adressent à des médecins et à des psychiatres, rédigent des comptes rendus et des lettres à leurs correspondants. S'ils étaient si inefficaces ou dangereux (en se passant de l'évaluation médicale), le nombre de plaintes gênerait la profession. Cela n'est pas le cas d'une part parce que de nombreuses demandes de psychothérapies ne sont pas directement médicales (ce qui ne signifie pas qu'elles ne soient pas graves, comme dans le cas des conflits familiaux, des difficultés scolaires, de la souffrance au travail), mais d'autre part parce que la tendance actuelle des patients est aussi de se rendre directement chez un psychologue pour y trouver aide, écoute et soutien.

\section{Une absence de conciliation (à la Française) des principaux interlocuteurs concernés}

Le dispositif proposé par la Sécurité sociale fait l'objet de plusieurs critiques. Le Syndicat national des psychologues en association avec la CGT (Santé, médecins, cadres, ingénieurs et techniciens), la FFPP (Fédération française des psychologues et de psychologie) et le SIUEERPP (Séminaire interuniversitaire d'enseignement et de recherche en psychopathologie et psychanalyse) ont écrit le 5 décembre 2018 une déclaration commune ${ }^{5}$. Cette déclaration demande prioritairement un renforcement du service public en psychiatrie et en psychologie. Elle s'appuie également sur les expériences du Royaume-Uni pour montrer que le passage par le médecin généraliste n'est pas le meilleur moyen d'adresser des patients en psychothérapie [3] et que $30 \%$ des patients choisissent eux-mêmes leur modalité psychothérapeutique sans en avoir jamais parlé à leur médecin généraliste [4] ; enfin, elle demande la reconnaissance du code de déontologie des psychologues qui déclare que les psychologues sont formés pour établir un diagnostic du fonctionnement psychique du patient, choisir les moyens de développer un bilan psychologique et le lui restituer et enfin proposer et réaliser une psychothérapie puis évaluer ses effets. En revanche, le médecin généraliste n'a, jusqu'à preuve du contraire, aucune formation à la psychothérapie. Rappelons qu'aujourd'hui le psychologue (et psychothérapeute) n'obtient ce titre qu'à l'issue de son master, adjoint à sa licence de psychologie et à ses stages d'un minimum de 500 heures. La proposition des syndicats se conclut par la possibilité d'un accès direct aux psychologues, sans passer par un médecin généraliste ou un psychiatre, et par la proposition directe de psychothérapie par le psychologue. La demande d'une concertation urgente est réclamée, car, en effet, la demande de psychothérapie est en augmentation. Notons que l'AFTCC était revenue en

\footnotetext{
${ }_{5}^{5}$ Expérimentation par la CNAM du remboursement des consultations auprès des psychologues. Site du Syndicat national des psychologues, le 5 décembre 2018.
} 
arrière dès avril 2018, par rapport à sa participation initiale et solipsiste à l'expérimentation. Sur le site de l'AFTCC, on trouve une déclaration qui regrette la dévalorisation de l'acte de psychothérapie, l'absence de prise en compte des qualifications du psychologue et psychothérapeute de se " prescrire » lui-même ; enfin, elle estime que la formation continue des psychothérapeutes est négligée dans la faiblesse du remboursement et que l'intervention de la Sécurité sociale comme tiers dans la relation patient-thérapeute forme un biais dans l'alliance thérapeutique [5].

Ce dispositif pose bien évidemment la question de la durée d'une psychothérapie, de la place du symptôme, de la prescription du soin psychique par le système médical avec des contraintes qui ne respectent pas la temporalité psychique. Nous avons demandé, lors d'une réunion avec l'ARS, quel serait le mode d'évaluation de ce dispositif au bout des trois ans, il nous a été répondu que cette évaluation serait effectuée par des économistes. Quel décalage avec l'essence de nos pratiques illustrée par l'ensemble des articles de ce numéro !

Évaluer les psychothérapies, ce n'est donc pas réitérer les effets d'un énième rapport à l'instar de la polémique créée par l'Inserm en 2004, mais élargir la vision du soin psychique. Cette dynamique repose sur la mise en lumière des expérimentations des uns et des autres, du développement d'outils thérapeutiques pertinents et évalués, et de la formation continue des praticiens. Un des enjeux majeurs de la psycho-oncologie est d'accompagner l'évolution des sociétés postmodernes tout en questionnant les innovations, pour le mieux-être des patients.

\section{Références}

1. Despland JN, de Roten Y, Kramer U (2018) L'évaluation des psychothérapies. Lavoisier, Paris, 246 p

2. Haute Autorité de santé (2017) Épisode dépressif caractérisé de l'adulte : prise en charge en soins de premier recours. Haute Autorité de santé, $\mathrm{p} 45$

3. Bebbington P, Meltzer H, Brugha T, et al (2003) Unequal access and unmet need: neurotic disorders and the use of primary care services. Int Rev Psychiatry 15:115-22

4. Brown JSL, Boardman J, Elliott SA, et al (2005) Are self-referrers just the worried well? - A cross-sectional study of self-referrers to community psycho-educational Stress and Self-Confidence workshops. Soc Psychiatry Psychiatr Epidemiol. 40:396-401

5. Expérimentation du remboursement des psychothérapies | AFTCC [Internet]. [Cité 30 décembre 2018]. Disponible sur : https://www. aftcc.org/experimentation-du-remboursement-des-psychotherapies 